\title{
ERRATA
}

\section{Erratum: Spin liquid in an almost ferromagnetic Kondo lattice [JETP 85, 399-414}

(August 1997)]

K. A. Kikoin, M. N. Kiselev, and A. S. Mishchenko

Kurchatov Institute, 123182 Moscow, Russia

[S1063-7761(97)02512-2]

The correct title of this paper should read as follows: "Spin liquid in an almost antiferromagnetic Kondo lattice.'

\section{Erratum: Inelastic light scattering by electrons and plasmons in metals [JETP 85, 370-375 (August 1997)]}

L. A. Falkovsky

L. D. Landau Institute for Theoretical Physics, Russian Academy of Sciences, 117334 Moscow, Russia

S. Klama

Institute of Molecular Physics, Polish Academy of Sciences, Smoluchowskiego 17, 60-179 Poznan, Poland

Zh. Éksp. Teor. Fiz. 112, 2263 (December 1997)

[S1063-7761(97)02612-7]

At the end of section "5. CONCLUSIONS" the following sentence should be added: "The other (S.K.) acknowledges the financial support of the Polish Research Committee of Sciences (Grant No. 2 P03B 101 12).', 